\title{
ARTICLE Motivation and sensitivity to monetary reward in late-life insomnia: moderating role of sex and the inflammatory marker CRP
}

\author{
Chloe C. Boyle (D) ${ }^{1}$, Joshua H. Cho (D) ${ }^{1}$, Naomi I. Eisenberger ${ }^{2}$, Richard E. Olmstead ${ }^{1}$, Dominique Piber ${ }^{1,3}$, Nina Sadeghi ${ }^{1}$, \\ Masih Tazhibi ${ }^{2}$ and Michael R. Irwin (D) ${ }^{1}$
}

\begin{abstract}
Insomnia is a well-established risk factor for late-life depression, yet the intermediary mechanisms are not known. One plausible mechanism is dysregulation of the reward system, a common feature of depression. The main objective of the current study was to determine whether late-life insomnia is associated with reduced motivation and reduced sensitivity for monetary reward. Secondary exploratory objectives were to test for sex-specific effects and whether elevated inflammation potentiated these associations. Nondepressed community dwelling older adults $(n=104$; aged $60-80)$ who either met $(n=31)$ or did not meet $(n=$ 73) criteria for insomnia disorder as assessed by the Structured Clinical Interview for DSM-5 completed the Effort Expenditure for Rewards Task and provided blood samples for the assessment of C-reactive protein (CRP). Older adults with late-life insomnia showed reduced reward motivation $95 \% \mathrm{Cl}[-0.955,-0.569]$ and reduced reward sensitivity $95 \% \mathrm{Cl}[-0.430,-0.075]$ relative to comparison controls. In secondary exploratory analyses, late-life insomnia was associated with reduced motivation to a greater degree in males than in females $95 \% \mathrm{Cl}[0.072,0.775]$, particularly when CRP was also elevated $95 \% \mathrm{Cl}[0.672,1.551]$. Late-life insomnia is associated with reduced motivation and sensitivity for monetary reward, which suggests insomnia may confer risk for late-life depression by dysregulation of reward mechanisms. Exploratory analyses suggest that older males with insomnia and elevated CRP may be particularly vulnerable to deficits in reward motivation. Although in need of replication and further study, results suggest that interventions that target insomnia or deficits in reward processing may mitigate the risk of depression in nondepressed older adults, especially older males with insomnia.
\end{abstract}

Neuropsychopharmacology (2020) 45:1664-1671; https://doi.org/10.1038/s41386-020-0735-7

\section{INTRODUCTION}

Insomnia is an established risk factor for depression onset, maintenance, and recurrence which becomes more prevalent with age [1-4]. Indeed, over one-third of older adults suffer from insomnia, and late-life insomnia has been shown to prospectively predict incident depressive symptoms and major depressive disorder [2,5]. In a large multi-site study, the continued presence of insomnia predicted the maintenance of depression in older adults over a 12-month period, and this was only partially mitigated by active antidepressant treatment [3]. Furthermore, sleep disturbance has been shown to predict depression recurrence in older adults independent of depressive symptom severity, chronic illness, or antidepressant use [6]. The mechanisms that contribute to the link between insomnia and depression are not known, and the absence of research is particularly striking in older adults given their prevalence of insomnia and the adverse effects of late-life depression on psychosocial and physical health $[1,7,8]$.

Insomnia may increase risk for late-life depression through dysregulation in the reward system. Reward dysregulation is not only a common feature of depression [9], but is also evident in nondepressed at-risk populations [10, 11] and prospectively predicts onset of depression and increases in depressive symptoms $[12,13]$. Anhedonia, a cardinal symptom of depression, is thought to be due to alterations in reward processing and can manifest as reduced motivation for reward (i.e., reduced "wanting"), reduced sensitivity for reward (i.e., reduced "liking"), and/or blunted reward learning. Older adults report difficulties with motivation in the context of depression [7] and are hypothesized to be vulnerable to reward dysregulation due to age-related decreases in frontostriatal dopaminergic function [14, 15].

In striking contrast to the prevalence of insomnia in older adults [1], as well as evidence of reward dysregulation in older adults $[14,15]$, no study to our knowledge has examined whether insomnia might contribute to changes in reward processing in older adults, although some research in adults supports this emerging hypothesis $[16,17]$. One small study found that adults with sleep complaints had lower desire for (i.e., "wanting") and enjoyment of (i.e., "liking") rewarding experiences than comparison controls over 7 days of self-report experience sampling [18]. Other work found that diagnostic insomnia is associated with reduced gray matter volume $[19,20]$ and altered functional

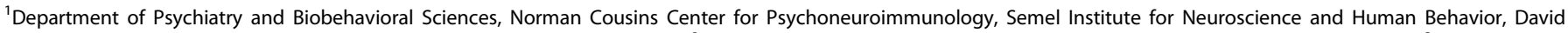

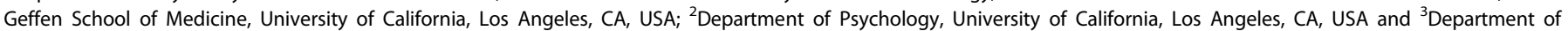
Psychiatry, Charité-Universitätsmedizin Berlin, Campus Benjamin Franklin, Berlin, Germany

Correspondence: Michael R. Irwin (mirwin1@ucla.edu)

Received: 6 February 2020 Revised: 13 May 2020 Accepted: 8 June 2020

Published online: 16 June 2020 
connectivity [21] in reward-related brain regions, although assessment of reward processes was not performed. It is not known whether insomnia as diagnosed by the Diagnostic and Statistical Manual (DSM)-5 [22] is associated with behavioral deficits in reward processing.

Furthermore, no studies have evaluated individual difference factors, such as sex and inflammatory burden, which could modulate effects of late-life insomnia on the reward system. Inflammation is implicated in depression pathophysiology, associated with alterations in reward function [23, 24], and elevated following sleep disturbance [25]. Moreover, inflammation predicts treatment response to antidepressants [24] and electroconvulsive therapy [26] as well as susceptibility to stress in preclinical models [27]. It is not known if elevated inflammation confers enhanced vulnerability to reward dysregulation in the context of late-life insomnia. Sex-specific effects in relation to insomnia have received little empirical attention, although adult females have been shown to exhibit lower motivation for monetary reward as compared to males [28], as well as greater alterations in reward processing following an inflammatory challenge [29].

To address these gaps, community dwelling older adults with and without late-life insomnia underwent evaluation of reward processing by administration of a standard monetary reward task, which assessed both reward motivation (i.e., "wanting") and reward sensitivity (i.e., "liking"). It is hypothesized that, in comparison to older adults without insomnia, those with late-life insomnia will exhibit (1) reduced reward motivation; and (2) reduced reward sensitivity. Secondary study objectives are to test for sex-specific effects and whether elevated inflammation potentiates these associations.

\section{MATERIALS AND METHODS}

Participants and procedure

Data for the current study were obtained from the baseline assessment of an ongoing randomized controlled trial investigating psychobiological effects of an acute inflammatory challenge (R01\# AG051944; Sleep and Healthy Aging Research on Depression Study; NCT03256760). All study procedures were approved by the University of California (UCLA) Institutional Review Board. Baseline assessment included administration of the Structured Clinical Interview for DSM-5 (SCID-DSM-5) by trained research staff and completion of self-report questionnaires and behavioral tasks. Participants were 104 healthy older adults (aged 60-79) who either met ( $n=31 ; 21$ female) or did not meet ( $n=73 ; 31$ female) criteria for insomnia disorder (i.e., late-life insomnia) as assessed by the SCID-DSM-5. Exclusion criteria are described in detail in Supplementary Material and included among others current psychiatric illness as assessed by the DSM- 5 except insomnia disorder; cognitive impairment as indicated by a score $<24$ on the Mini-Mental Status Exam (MMSE) ${ }^{1}$; sleep apnea as confirmed by polysomnography; presence of an acute infectious illness in the 2 weeks prior to an experimental session (via self-report and white blood cell count within normal range); $\mathrm{BMI}>35 \mathrm{~kg} / \mathrm{m}^{2}$; smoking; use of medications known to modulate the immune system; use of over the counter or prescription sleep aids (via self-report).

Participants were recruited from the Los Angeles community August 2016-July 2019 using the GENESYS Sampling Systems company, which provided contact information for households with at least one person aged 60 years or older within a 10 mile radius of UCLA. A total of 283 responded to recruitment letters and phone calls and completed phone and in-person eligibility screening. Out of 117 eligible participants who provided Creactive protein (CRP) data, 115 provided complete self-report

\footnotetext{
${ }^{1}$ All participants scored $>28$ on the MMSE, and inclusion of MMSE scores as a covariate did not alter results.
}

questionnaire data and 113 completed the Effort Expenditure for Rewards Task (EEfRT) (data lost by computer malfunction $(n=4)$ ). Six participants were excluded for completing fewer than 18 trials on the EEfRT, and one participant was excluded due to levels of CRP at $36.3 \mathrm{mg} / \mathrm{L}$. In total, 104 participants provided complete data across all measures; participants with missing data did not differ from participants with full data in terms of insomnia diagnosis or demographic or psychosocial characteristics (Table S2).

\section{Assessments}

Insomnia and related behavioral symptoms. Insomnia disorder was diagnosed by SCID-DSM-5 and confirmed in a diagnostic consensus meeting with a board-certified psychiatrist (MRI). Severity of insomnia symptoms was assessed with the Insomnia Severity Index. Severity of depressive symptoms was assessed by the Beck Depression Inventory-Il; the scale item relating to sleep quality was removed prior to calculating the total score. Physical fatigue symptoms were assessed with the physical fatigue subscale from the Multidimensional Fatigue Inventory Scale. Depressive and fatigue symptoms were assessed and included as covariates in statistical analyses to ensure they were not contributing to possible group differences in reward processing; both have been associated with reward motivation [30, 31] and insomnia [32].

Behavioral reward task. The EEfRT [28] was used to evaluate reward processing. The EEfRT is a computerized task that assesses effort-based decision making in the context of monetary reward. During the task, participants are presented with a series of trials in which they choose between an easy, low-effort trial (worth a low reward amount of \$1.00) and a hard, high-effort trial (worth higher reward amounts ranging between \$1.24 and \$4.30). Easy trials required 30 button presses using the index finger of the nondominant hand in $7 \mathrm{~s}$, while hard trials required 100 button presses with the pinky finger of the dominant hand in $21 \mathrm{~s}$. Participants were told that not all successfully completed trials would be rewarded, and the probability that a successful response would yield a reward $(12,50$, and $88 \%)$ was presented for each trial. In the current study, the EEfRT was shortened from 20 to 10 min and hard trials used the pinky finger of the dominant hand rather than the nondominant hand to accommodate constraints in the laboratory environment (also used in [23]).

Motivation for reward on the EEfRT is operationalized by willingness to exert effort for monetary reward; i.e., the selection of high-effort/high-reward trials relative to the selection of loweffort/low-reward trials. Sensitivity to reward is operationalized by the association between changes in monetary reward magnitude (ranging from \$1.24 to \$4.30) and changes in likelihood of selecting high-effort/high-reward trials vs. low-effort/low-reward trials $[23,33]$.

Inflammation. Inflammation was assessed by measuring circulating levels of the systemic inflammatory marker CRP between the hours of 8 and 9 a.m. Participants provided blood samples prior to receiving breakfast as part of the larger experimental protocol, but were not required to fast. Blood samples were collected by venipuncture into EDTA tubes, placed on ice, centrifuged for acquisition of plasma, and stored at -80 for batch testing. CRP levels were determined utilizing the Human CRP Quantikine ELISA (R\&D Systems) according to the manufacturer's protocol with a lower limit of detection of $0.2 \mathrm{mg} / \mathrm{L}$. Samples were assayed in duplicate. Intra- and inter-assay precision of all tests was $<6.1 \%$.

\section{Sample size}

No studies have evaluated group differences in effort-based decision making in association with insomnia disorder. Several studies have evaluated EEfRT performance in other patient 
populations relative to comparison control participants and yielded large effect sizes $(d>0.8)$ [30,31]. Assuming an effect size of $d=0.8,100$ participants in the current study provide statistical power of $>90 \%(a=0.05)$ to detect differences in EEfRT performance as a function of insomnia, the primary objective of the current study. For secondary analyses, past studies have shown small to medium effects of sex $[23,28]$ and inflammation [23] on EEfRT performance. Given these estimates, we recognize that secondary analyses that examine moderating effects of sex or inflammation on reward processing as a function of insomnia are exploratory, as they are not adequately powered.

Analytic approach

Data reduction. Participants who chose fewer than 18 total trials were excluded ( $n=6 ; 5$ comparison controls). Individual EEfRT trials in which the participant did not choose between an easy or hard task were excluded $(7.28 \%$ of all trials). Participants successfully completed $93.94 \%$ of the hard trials and $82.46 \%$ of the easy trials; trials that the participant selected but completed fewer than $10 \%$ of the required button presses were excluded ( $n$ $=39$ trials; $0.96 \%$ of all trials).

Generalized estimating equations (GEE) models. GEE with a binary logistic model and independent working correlation structure were used to analyze decision-making behavior on the EEfRT; this is a typical approach for this task as GEE account for correlated data and are appropriate for a binary dependent variable [28]. The working correlation structure was determined with the STATA program QIC (quasilikelihood under the independence model criterion).

Consistent with previous studies [28], the following task-specific variables were included as continuous time-varying covariates: reward magnitude (range \$1.24-\$4.30), probability, and, to control for fatigue effects during the task, trial number (see Table S1 for GEE models with these task-specific variables only). Betweensubject predictors were group (insomnia vs. control), severity of depressive symptoms, severity of physical fatigue, and sex. The dependent binary variable was coded as 0 (low-effort/low-reward choice) and 1 (high-effort/high-reward choice). Level of significance was set at $p<0.05$ and hypothesis tests were two sided.

Assessment of reward motivation. Motivation for reward was operationalized as willingness to exert effort for a monetary reward. Hence, reduced reward motivation was characterized as being less willing to exert effort for a higher amount of monetary reward (i.e., choosing fewer high-effort/high-reward trials and more low-effort/low-reward trials). The predictor of interest for the primary analysis was the group (insomnia vs. control) variable. For sex-specific analyses, sex differences in reward motivation were tested as the main effect of sex in the prediction of willingness to exert effort for a high monetary reward. Sex differences in relation to insomnia were tested with a two-way interaction term (sex by group).

Assessment of reward sensitivity. Sensitivity to reward was operationalized by the association between changes in monetary reward magnitude and changes in likelihood of selecting higheffort/high-reward trials vs. low-effort/low-reward trials [23, 33]. Hence, reduced reward sensitivity was represented by an attenuated association between increases in potential monetary reward magnitude and increased likelihood of selecting higheffort/high-reward trials. Late-life insomnia vs. comparison control group differences in reward sensitivity were tested using an interaction term between group and reward magnitude. For sexspecific analyses, sex differences in reward sensitivity were tested with an interaction term between sex and reward magnitude; this allowed us to ascertain whether changes in monetary reward magnitude were associated with changes in likelihood of selecting high-effort/high-reward trials differently as a function of sex. Sex differences in relation to late-life insomnia for reward sensitivity were tested with a three-way interaction term (sex by reward magnitude by group).

Assessment of CRP in late-life insomnia and in association with EEfRT performance. Levels of CRP were evaluated for outliers and due to positive skew were log transformed prior to analyses; seven participants with levels below the limit of detection were imputed to $50 \%$ of the lower limit of detection; one participant was excluded as an outlier (CRP of $36.3 \mathrm{mg} / \mathrm{L}$ ) and all other CRP values were within 3 standard deviations of the mean. To evaluate CRP as a moderator of the effect of late-life insomnia on reward motivation, a two-way interaction term between group (binary) and CRP (continuous) was tested in the GEE model predicting hard trial choice. Sex-specific analyses included a three-way interaction term (sex by group by CRP). To evaluate CRP as a moderator of the effect of late-life insomnia on reward sensitivity, a three-way interaction term (group by reward magnitude by CRP) was tested. Sex-specific analyses included a four-way interaction term (sex by group by reward magnitude by (RP). Models with CRP controlled for age, BMI, and ethnicity.

\section{RESULTS}

Participant characteristics

Participants were community dwelling older adults $\left(M_{\text {age }}=65.86\right)$ with $(n=31)$ and without $(n=73)$ late-life insomnia who were predominantly white, educated, and of normal BMI (Table 1). There were more females than males in the insomnia group $(n=$ 21 females) but not in the comparison control group $(n=31$ females). The groups did not otherwise differ on sociodemographic characteristics (Table 1). Older adults with late-life insomnia had significantly higher severity of insomnia, depressive, and physical fatigue symptoms, as compared to comparison controls (Table 1), with no further differences as a function of sex (see Table S3 for differences by sex collapsing across groups).

Levels of CRP were on average low $(M=1.73 \mathrm{mg} / \mathrm{L}, \mathrm{SD}=2.38$; range: $0.1-14.55 \mathrm{mg} / \mathrm{L})$ and did not differ by sex $(p=.761)$. CRP was significantly elevated in individuals with late-life insomnia relative to comparison controls, $t(114)=2.040, p=.044, d=.413$, and CRP was correlated with severity of insomnia symptoms, $r=$ $0.280, p=0.002$, for all participants with CRP data. Similar patterns were evident for the subset of participants with full data across all measures, $t(102)=1.600, p=0.113, d=0.343, r=0.292, p=0.002$.

\section{Reward motivation}

Late-life insomnia was hypothesized to be associated with reduced reward motivation. Indeed, results showed that those with late-life insomnia were significantly less likely to select hard (high effort/high reward) trials than comparison controls $(b=$ $-0.580, \mathrm{SE}=0.078, p<0.001 ; 95 \% \mathrm{Cl}[-0.732,-0.428])$. This association remained significant controlling for sex, depressive and physical fatigue symptoms, and task-specific variables (i.e., reward magnitude, probability, and trial number) $(95 \% \mathrm{Cl}[-0.955$, -0.569 ]; Table 2; Fig. 1). To further interrogate the role of insomnia on reward motivation, we examined whether severity of insomnia symptoms was associated with hard trial choice across the sample, and found that more severe insomnia symptoms predicted fewer hard trial choices in unadjusted $(95 \% \mathrm{Cl}[-0.026,-0.0004])$ and adjusted $(95 \% \mathrm{Cl}[-0.048,-0.013])$ models (Table S4). Thus, both insomnia diagnosis and severity of insomnia in older adults were associated with a shift away from high-effort/high-reward trials and toward low-effort/low-reward trials, indicative of reduced reward motivation for monetary reward in the context of effortbased decision making. 


\begin{tabular}{|lcc|}
\hline Table 1. Demographic and psychosocial characteristics of community dwelling older adults with and without late-life insomnia. \\
\hline Variable $^{\text {a }}$ & $\begin{array}{l}\text { Older adults with late-life insomnia } \\
(n=31)\end{array}$ & $\begin{array}{l}\text { Older adults without late-life insomnia } \\
(n=73)\end{array}$ \\
\hline Age, mean years (SD) & $65.71(4.25)$ & $65.92(4.51)$ \\
Female sex*, $n(\%)$ & $21(68 \%)$ & $31(42 \%)$ \\
BMI, mean (SD) & $25.06(3.13)$ & $24.76(3.13)$ \\
CRP, mean (SD) & $2.03(2.49)$ & $1.60(2.35)$ \\
White ethnicity, $n(\%)$ & $24(77 \%)$ & $58(79 \%)$ \\
Education, bachelor's degree and higher, $n(\%)$ & $23(76.67 \%)$ & $50(69 \%)$ \\
Depressive symptoms**, mean (SD) & $5.84(4.92)$ & $1.84(2.04)$ \\
History of diagnosed depression, $n$ (\%) & $8(26 \%)$ & $8(11 \%)$ \\
Physical fatigue symptoms*, mean (SD) & $1.98(2.33)$ & $1.12(1.33)$ \\
Insomnia symptom severity**, mean (SD) & $11.35(5.52)$ & $1.24(1.62)$ \\
\hline Depressive symptoms were assessed with the Beck Depression Inventory-ll; the scale item relating to sleep quality was removed prior to calculating the total \\
score. Physical fatigue symptoms were assessed with the physical fatigue subscale from the Multidimensional Fatigue Inventory Scale. Severity of insomnia \\
symptoms was assessed with the Insomnia Severity Index.
\end{tabular}

Table 2. Reward motivation is reduced in older adults with late-life insomnia relative to comparison controls (Model 1) with sex-specific effects (Model 2) using Generalized Estimating Equations (GEE) and adjusting for participant characteristics and task-specific variables. Reward sensitivity is reduced in those with late-life insomnia relative to comparison controls (Model 3) with no sex-specific effects (Model 4).

\begin{tabular}{|c|c|c|c|c|c|c|c|c|}
\hline Variable & $\mathrm{b}(\mathrm{SE})$ & $z$ & $\mathrm{~b}(\mathrm{SE})$ & z & $\mathrm{b}(\mathrm{SE})$ & z & $\mathrm{b}(\mathrm{SE})$ & z \\
\hline Group & $-0.762(0.096)^{* *}$ & -7.74 & $-1.134(0.152)^{* *}$ & -7.46 & $-0.089(0.259)$ & -0.34 & $-1.197(0.440)^{*}$ & -2.72 \\
\hline Sex & $-0.292(0.079)^{* *}$ & -3.68 & $0.113(0.148)$ & 0.76 & $-0.293(0.080)^{* *}$ & -3.68 & $1.319(0.462)^{*}$ & 2.86 \\
\hline Depressive symptoms & $0.069(0.012)^{* * *}$ & 5.51 & $0.072(0.012)^{* *}$ & 5.76 & $0.067(0.012)^{* * *}$ & 5.44 & $0.072(0.012)^{* *}$ & 5.79 \\
\hline Probability & $0.181(0.048)^{* * *}$ & 3.79 & $0.182(0.048)^{* *}$ & 3.81 & $0.181(0.048)^{* * *}$ & 3.80 & $0.182(0.048)^{* *}$ & 3.81 \\
\hline Trial number & $-0.041(0.004)^{* *}$ & -10.28 & $-0.041(0.004)^{* *}$ & -10.29 & $-0.041(0.004)^{* * *}$ & -10.26 & $-0.041(0.004)^{* *}$ & -10.25 \\
\hline Group $\times$ sex & & & $-0.570(0.176)^{* *}$ & -3.24 & & & $-1.555(0.544)^{*}$ & -2.86 \\
\hline Group $\times$ reward magnitude & & & & & $-0.253(0.091)^{*}$ & -2.79 & $0.016(0.155)$ & 0.10 \\
\hline Sex $\times$ reward magnitude & & & & & & & $-0.089(0.106)$ & -0.83 \\
\hline
\end{tabular}

Sex differences and reward motivation. Female sex was associated with reduced reward motivation, i.e., lower likelihood of selecting hard trials, in comparison to male sex, as indicated by a main effect of sex in unadjusted $(b=-0.421, \mathrm{SE}=0.078, p<0.001 ; 95 \%$ $\mathrm{Cl}[-0.564,-0.278])$ and adjusted $(95 \% \mathrm{Cl}[-0.448,-0.137] ;$ Table 2) models. To examine whether this sex difference further varied as a function of insomnia status, a two-way interaction term between sex and insomnia group was examined. The interaction between sex and insomnia was significant, such that late-life insomnia was associated with a reduction in selection of hard trials to a greater degree in males as compared to females in unadjusted $(b=0.390, \mathrm{SE}=0.163, p=0.017 ; 95 \% \mathrm{Cl}[0.070$,
0.710]) and adjusted (95\% Cl $[-0.225,-0.915]$; Table 2) models (Fig. 1). Follow-up within-group analyses found that both males $(b=-0.248, \mathrm{SE}=0.033, p<0.001)$ and females $(\mathrm{b}=-0.127, \mathrm{SE}=$ $0.026, p<0.001$ ) exhibited a reduction in monetary reward motivation in association with late-life insomnia, and this difference was significantly greater for males, $X^{2}(1)=9.97, p=$ 0.002 .

Inflammation and reward motivation. There was no evidence that elevated systemic inflammation accentuated the effect of late-life insomnia on reward motivation in adjusted or unadjusted models ( $p^{\prime}$ s > 0.7; Fig. 2). However, sex-specific analyses revealed a 

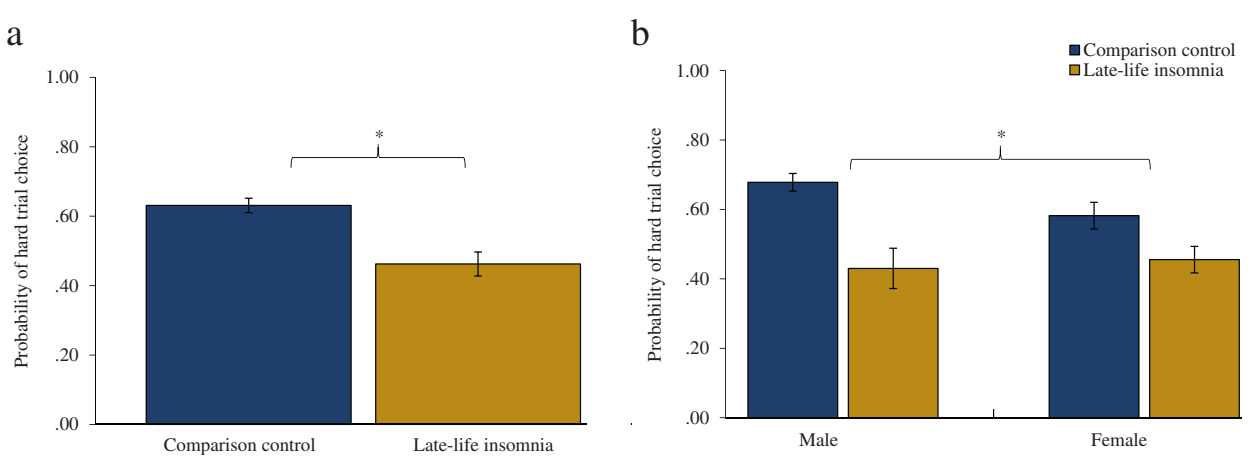

Fig. 1 Reward motivation in relation to late-life insomnia and sex plotted with $\mathbf{9 5 \%}$ confidence intervals and adjusted for participant and task characteristics. Reward motivation is significantly reduced in older adults with late-life insomnia $(n=31 ; 21$ female) relative to comparison controls $(n=73 ; 31$ female) $(p<0.001$; (a)) with evidence of sex-specific effects $(p=0.001$; (b)). Late-life insomnia is associated with reduced reward motivation in males $(p<0.001)$ and females $(p<0.001)$, and this reduction is greater for males than females $(p=0.002)$.

a

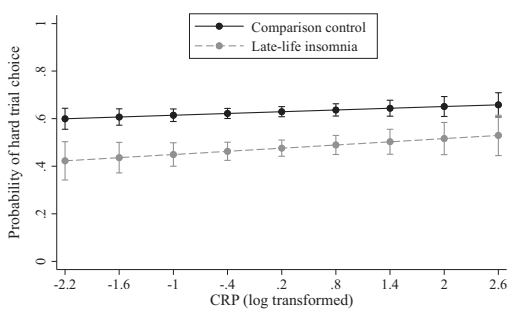

b

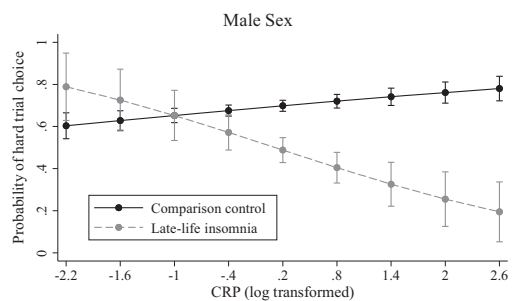

c

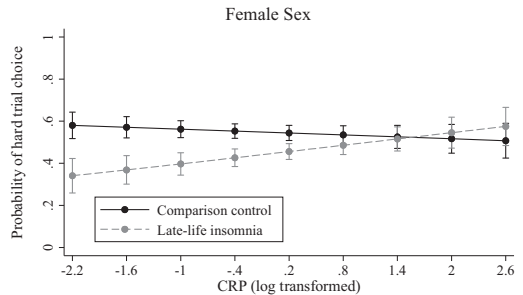

Fig. 2 Reward motivation in relation to late-life insomnia, sex, and systemic inflammation plotted with $95 \%$ confidence intervals and adjusted for participant and task characteristics. CRP is not related to reward motivation among older adults with late-life insomnia ( $n=31$; 21 female) or comparison controls across sexes $(n=73 ; 31$ female) $(p=0.614$; (a)) but there were sex-specific effects ( $p<0.001)$. Among males with late-life insomnia, higher levels of CRP are associated with lower reward motivation $(p<0.001 ;(\mathbf{b}))$. CRP was not significantly associated with reward motivation for females with or without late-life insomnia $\left(p^{\prime} s>0.1 ;(\mathbf{c})\right)$.

significant three-way interaction $(b=1.091, \mathrm{SE}=0.214, p<0.001$; $95 \% \mathrm{Cl}[0.672,1.551])$, such that among males only, higher CRP was associated with lower monetary reward motivation for those with late-life insomnia relative to comparison controls. This association held in models adjusted for depressive and fatigue symptoms, task-specific variable, age, BMl, and ethnicity $(b=$ 1.131, SE $=0.241, p<0.001 ; 95 \% \mathrm{Cl}[0.658,1.604]$; Table S5). As shown in Fig. 2 and confirmed by follow-up within-group analyses, higher levels of CRP were associated with a reduction in monetary reward motivation for males with late-life insomnia $(b=-0.150$, $\mathrm{SE}=0.041, p<0.001)$ but not comparison control males $(b=$ $0.024, \mathrm{SE}=0.017, p=0.142)$, comparison control females $(b=$ $-0.027, \mathrm{SE}=0.018, p=0.130$ ), or females with late-life insomnia $(b=0.035, \mathrm{SE}=0.023, p=0.118)$.

\section{Reward sensitivity}

Late-life insomnia was also hypothesized to be associated with reduced sensitivity to reward. Consistent with hypotheses, those with late-life insomnia showed an attenuated association between increases in potential monetary reward and increases in hard trial choice, as compared to comparison controls $(b=-0.265$, SE $=$ $0.087, p=0.002 ; 95 \% \mathrm{Cl}[-0.436,-0.094])$. Results remained significant in an adjusted model (95\% Cl [-0.430, -0.075]; Table 2). Follow-up within-group analyses confirmed that while increases in reward magnitude were associated with increases in hard trial choice for both those with $(b=0.079, \mathrm{SE}=0.016, p<$ $0.001)$ and without $(b=0.128, \mathrm{SE}=0.010, p<0.001)$ late-life insomnia, this association was significantly attenuated in those with late-life insomnia as compared to comparison controls, $X^{2}(1)$ $=6.72, p=0.010$ (Fig. 3). There was no evidence that higher insomnia symptom severity across the sample was associated with reduced reward sensitivity $(p>0.4)$.
Sex differences and reward sensitivity. Sex was associated with reward sensitivity, as represented by a significant interaction between sex and reward magnitude in the prediction of hard trial choice in unadjusted $(b=-0.227, \mathrm{SE}=0.083, p=0.006 ; 95 \% \mathrm{Cl}[-0.389$, $-0.064])$ and adjusted $(b=-0.226, \mathrm{SE}=0.086, p=0.008 ; 95 \% \mathrm{Cl}$ $[-0.394,-0.058]$; Table S6) models. Follow-up within-group analyses found that while increases in reward magnitude were significantly associated with increases in hard trial choice for both male $(b=0.132$; $\mathrm{SE}=0.012, p<0.001)$ and female $(b=0.094 ; \mathrm{SE}=0.013, p<0.001)$ older adults, this effect was significantly stronger for males $X^{2}(1)=$ $5.07, p=0.024$. Thus, male sex in older adults is associated with higher sensitivity to monetary reward on the EEfRT. There was no evidence that this sex difference varied as a function of insomnia, as tested by a three-way interaction term (group by reward magnitude by sex) ( $p=0.063 ; 95 \% \mathrm{Cl}[-0.743,0.019]$; Table 2, Fig. 3).

Inflammation and reward sensitivity. There was no evidence that elevated inflammation moderated the effect of late-life insomnia on reward sensitivity $(b=0.206, \mathrm{SE}=0.121, p=0.089 ; 95 \% \mathrm{Cl}$ $[-0.032,0.444])$, nor was there evidence for further moderation by sex $(b=-0.456, \mathrm{SE}=0.263, p=0.084 ; 95 \% \mathrm{Cl}[-0.972,0.061])$ in adjusted models.

\section{DISCUSSION}

Late-life insomnia is associated with reduced motivation and reduced sensitivity for monetary reward. As compared to community dwelling older adults without insomnia, those with late-life insomnia were less willing to exert effort to attain a higher monetary reward. Those with late-life insomnia were also less behaviorally responsive to increases in monetary reward, indicating a reduction in sensitivity to increases in reward magnitude. These effects were 
a

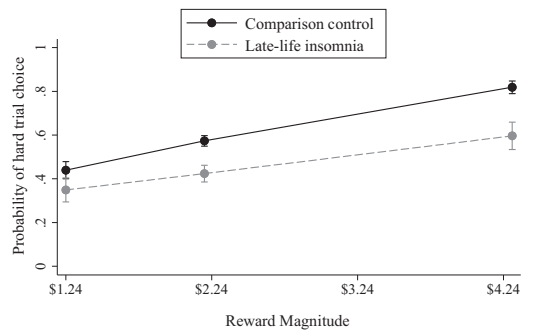

b

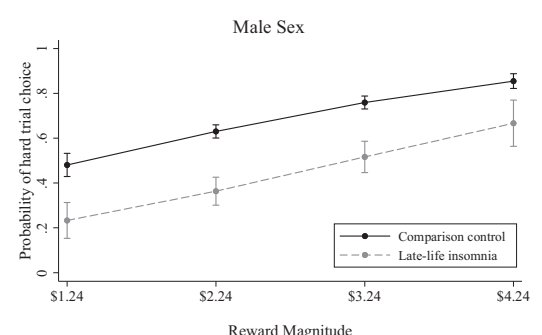

c

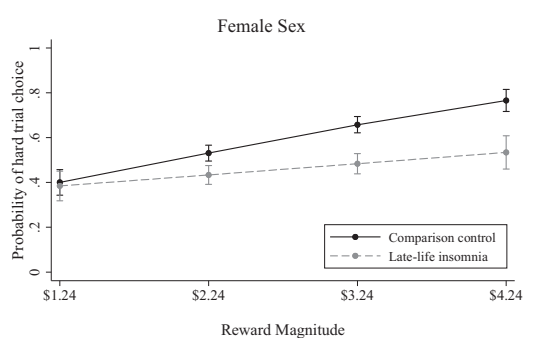

Fig. 3 Reward sensitivity in relation to late-life insomnia and sex plotted with $\mathbf{9 5 \%}$ confidence intervals and adjusted for participant and task characteristics. Older adults with late-life insomnia $(n=31 ; 21$ female) show reduced reward sensitivity relative to comparison controls $(n=73 ; 31$ female) $(p=0.005 ;(\mathbf{a}))$ with no evidence for sex-specific effects $(p=0.063 ;(\mathbf{b}, \mathbf{c}))$.

not attributable to group differences in depressive symptoms or fatigue, which underscores the importance of the reward system as a potential clinical target in efforts to decrease risk for late-life depression, particularly in the context of other risk factors such as insomnia. Moreover, results provide preliminary evidence that males may be more vulnerable to insomnia and inflammation-associated reductions in motivation for monetary reward.

There is an emerging literature linking sleep disturbance to dysregulated reward processing. One study found that adults with sleep complaints exhibited lower "wanting" for and "liking" of positive daily experiences than comparison controls [18], which parallels the insomnia-associated reductions in motivation and sensitivity observed in the current study. Consistent with these behavioral effects, structural and functional alterations in rewardrelated brain regions have been observed in adult insomnia patients relative to comparison controls $[20,21]$. In experimental studies, sleep deprivation alters reactivity in reward neurocircuitry in response to rewarding stimuli $[34,35]$, suggesting a causal role for sleep loss in reward dysregulation. Several studies have shown associations between sleep disturbance in adolescence and alterations in neural reactivity to reward or functional connectivity in corticolimbic reward circuitry [36,37]. Thus, although small, the literature converges in demonstrating an interconnection of the sleep and reward systems. The present study extends these prior findings and demonstrates an association between late-life insomnia and reward processing, using a validated assessment of multiple reward processes on a behavioral level.

Several lines of evidence support a role for inflammation in the association between late-life insomnia and reward processing. Treatment of insomnia has been shown to reduce systemic, cellular, or genomic markers of inflammation [38] and elevated inflammation can interfere with dopamine synthesis and function [24] and has been correlated with alterations in behavioral reward tasks [23] and reward neurocircuitry [39]. In the current study CRP was elevated in older adults with late-life insomnia, and among males elevated CRP accentuated the effect of late-life insomnia on reward motivation. This finding is consistent with other work demonstrating sex-specific associations between inflammation and depression or depressive phenotypes [40,41], but additional study is required to ascertain the determinants of the presence and direction of these associations.

Prior research has also shown sex differences in reward processing, with adult females exhibiting reduced motivation for monetary reward relative to adult males $[28,31]$. The current study suggests this pattern remains evident in older adults, despite the array of neurobiological and socioemotional changes that accompany aging [7, 14, 15, 42, 43]. However, in the presence of late-life insomnia, this pattern was reversed; males showed a heightened vulnerability to dysregulation of monetary reward processes, in which late-life insomnia conferred greater reductions in reward motivation in males relative to females. The fact that insomnia reverses sex difference in monetary reward processing is striking, especially given evidence of the male sex advantage in motivational processing in the older adult comparison controls. This enhanced vulnerability for males with insomnia was most marked in the context of elevated inflammation, an unexpected finding that is nevertheless consistent with large scale epidemiological studies linking elevated inflammation to depressive symptoms in males [41]. If corroborated by future studies, this finding would have clinical implications for precision medicine in psychiatry; specifically, older males with insomnia and elevated inflammation may preferentially benefit from interventions targeted at remediating reductions in reward motivation.

While speculative, the heightened salience of monetary reward for males in the current study may also be linked to key developmental transitions in aging. Retirement can increase risk for depression [44], with older males at elevated risk [45], possibly due to new challenges to self-worth, self-efficacy, and productivity that can be intertwined with monetary reward and perceived masculinity. Indeed, financial and occupational stressors have been shown to predict depression in males, whereas interpersonal social stressors predict depression in females [46]. Addressing these contextual socioemotional and environmental factors will be important in developing treatments to address reward dysregulation. Furthermore, the extent to which insomnia alters response to social rewards, and how this may vary by sex, requires further examination. Most notably, there is evidence for greater sensitivity to social reward in females as compared to males [47].

These results should be interpreted in the context of several limitations. As a cross-sectional observational study, no causal conclusions can be made. While validated and widely used, the EEfRT does not assess affective response to reward and uses monetary reward only; a fine-grained interrogation of the multifaceted reward system using multiple tasks and social rewards will enrich future studies. The EEfRT is also a complex decision-making task, and aging is most consistently associated with impaired reward processing for tasks requiring integration of reward information [48] rather than disruption of more basic reward function [49]. Whether insomnia disrupts basic reward function in older adults requires examination with less cognitively burdensome tasks. While the study had adequate statistical power to answer the primary study aims, secondary analyses on sex differences and CRP in relation to insomnia and reward sensitivity were exploratory, as they were not adequately powered. Moreover, the sample size for males with insomnia was small, and moderation analyses focusing on sex differences and CRP in relation to reward motivation should be interpreted with caution and require replication. Finally, although plasma CRP is a common indicator of systemic inflammation that predicts health outcomes and has been shown to correlate with other markers of inflammation, including inflammatory cytokines in both plasma and cerebrospinal fluid [50], assessment of a broader array of inflammatory biomarkers would provide a more comprehensive picture of immune system status. 
Insomnia is prevalent in older adults and a well-established risk factor for depression. Results from the current study suggest that dysregulation in the reward system may be one mechanism linking insomnia to late-life depression; if confirmed by future studies, this suggests that interventions that either target insomnia and/or deficits in reward processing may mitigate the risk of depression in nondepressed older adults, especially males with insomnia. Findings also indicate that late-life insomnia is associated with both reduced motivation and reduced reward sensitivity, which has further implications for treatment given that these constructs are phenomenologically and biologically distinguishable. An important topic moving forward is to identify whether males with elevated inflammation are especially vulnerable to insomnia-induced deficits in reward function, as our exploratory findings suggest. Ultimately, characterizing these mechanistic pathways is a critical step toward refined prevention and treatment of depression, particularly given that insomnia, elevated inflammation, and reward deficits are all modifiable risk factors that can be specifically targeted for intervention.

\section{FUNDING AND DISCLOSURE}

The authors declare no competing interests. This work was supported by the National Institutes of Health, USA (R01AG051944, R01AG056424-01, R01AG026364, R01CA160245, R01AG057750, and R01CA207130), the Norman Cousins Center for Psychoneuroimmunology (MRI), UCLA Clinical and Translational Science Institute (UL1TR001881), and Max Kade Foundation.

\section{AUTHOR CONTRIBUTIONS}

All authors provided final approval of the version of this article to be published. CCB, $\mathrm{NIE}, \mathrm{REO}$, and MRI substantially contributed to the conception and design of the work. CCB, NIE, REO, and MRI, as well as DP, NS, and MT, contributed to the acquisition, analysis, or interpretation of the work. CCB, NIE, JHC, and MRI contributed to the drafting the work and revising it critically for intellectual content. All authors are in agreement to be accountable for all aspects of the work in ensuring that questions related to the accuracy or integrity of any part of the work are appropriately investigated and resolved.

\section{ADDITIONAL INFORMATION}

Supplementary Information accompanies this paper at (https://doi.org/10.1038/ s41386-020-0735-7)

Publisher's note Springer Nature remains neutral with regard to jurisdictional claims in published maps and institutional affiliations.

\section{REFERENCES}

1. Stepnowsky CJ, Ancoli-Israel S. Sleep and its disorders in seniors. Sleep Med Clin. 2008;3:281-93.

2. Baglioni C, Battagliese G, Feige B, Spiegelhalder K, Nissen C, Voderholzer U, et al. Insomnia as a predictor of depression: a meta-analytic evaluation of longitudinal epidemiological studies. J Affect Disord. 2011;135:10-9.

3. Pigeon WR, Hegel M, Unützer J, Fan M-Y, Sateia MJ, Lyness JM, et al. Is insomnia a perpetuating factor for late-life depression in the IMPACT cohort? Sleep. 2008;31:481-8.

4. Li L, Wu C, Gan Y, Qu X, Lu Z. Insomnia and the risk of depression: a meta-analysis of prospective cohort studies. BMC Psychiatry. 2016;16:498.

5. Jaussent I, Bouyer J, Ancelin M-L, Akbaraly T, Peres K, Ritchie K, et al. Insomnia and daytime sleepiness are risk factors for depressive symptoms in the elderly. Sleep. 2011;34:1103-10.

6. Cho HJ, Lavretsky H, Olmstead R, Levin MJ, Oxman MN, Irwin MR. Sleep disturbance and depression recurrence in community-dwelling older adults: a prospective study. Am J Psychiatry. 2008;165:1543-50.

7. Power C, Greene E, Lawlor BA. Depression in late life: etiology, presentation, and management. Mental health and illness of the elderly. Singapore: Springer; 2017. p. 187-218.

8. Wei J, Hou R, Zhang X, Xu H, Xie L, Chandrasekar EK, et al. The association of latelife depression with all-cause and cardiovascular mortality among community- dwelling older adults: systematic review and meta-analysis. $\mathrm{Br} J$ Psychiatry. 2019;215:449-55.

9. Keren H, O'Callaghan G, Vidal-Ribas P, Buzzell GA, Brotman MA, Leibenluft E, et al. Reward processing in depression: a conceptual and meta-analytic review across fMRI and EEG studies. Am J Psychiatry. 2018;175:1111-20.

10. Gotlib IH, Hamilton JP, Cooney RE, Singh MK, Henry ML, Joormann J. Neural processing of reward and loss in girls at risk for major depression. Arch Gen Psychiatry. 2010;67:380.

11. McCabe C, Woffindale C, Harmer CJ, Cowen PJ. Neural processing of reward and punishment in young people at increased familial risk of depression. Biol Psychiatry. 2012;72:588-94.

12. Nelson BD, Perlman G, Klein DN, Kotov R, Hajcak G. Blunted neural response to rewards as a prospective predictor of the development of depression in adolescent girls. Am J Psychiatry. 2016;173:1223-30.

13. Stringaris A, Vidal-Ribas Belil $P$, Artiges $E$, Lemaitre $H$, Gollier-Briant F, Wolke $S$, et al. The brain's response to reward anticipation and depression in adolescence: dimensionality, specificity, and longitudinal predictions in a community-based sample. Am J Psychiatry. 2015;172:1215-23.

14. de Boer L, Axelsson J, Riklund K, Nyberg L, Dayan P, Bäckman L, et al. Attenuation of dopamine-modulated prefrontal value signals underlies probabilistic reward learning deficits in old age. Elife. 2017;5:95.

15. Dreher JC, Meyer-Lindenberg A, Kohn P, Berman KF. Age-related changes in midbrain dopaminergic regulation of the human reward system. Proc Natl Acad Sci. 2008;105:15106-11.

16. Boland EM, Bertulis K, Leong SH, Thase ME, Gehrman PR. Preliminary support for the role of reward relevant effort and chronotype in the depression/insomnia comorbidity. J Affect Disord. 2019;242:220-3.

17. Hasler BP, Germain A, Nofzinger EA, Kupfer DJ, Krafty RT, Rothenberger SD, et al. Chronotype and diurnal patterns of positive affect and affective neural circuitry in primary insomnia. J Sleep Res. 2012;21:515-26.

18. Lindert te BHW, Itzhacki J, van der Meijden WP, Kringelbach ML, Mendoza J, Van Someren EJW. Bright environmental light ameliorates deficient subjective "liking" in insomnia: an experience sampling study. Sleep. 2018;41:1-13.

19. Joo EY, Noh HJ, Kim J-S, Koo DL, Kim D, Hwang KJ, et al. Brain gray matter deficits in patients with chronic primary insomnia. Sleep. 2013;36:999-1007.

20. Altena E, Vrenken H, Van Der Werf YD, van den Heuvel OA, Van Someren EJW. Reduced orbitofrontal and parietal gray matter in chronic insomnia: a voxelbased morphometric study. Biol Psychiatry. 2010;67:182-5.

21. Wang L, Wang K, Liu J-H, Wang Y-P. Altered default mode and sensorimotor network connectivity with striatal subregions in primary insomnia: a resting-state multi-band fmri study. Front Neurosci. 2018;12:362.

22. Association AP. Diagnostic and statistical manual of mental disorders (DSM-5). American Psychiatric Publishing; Arlington, VA, 2013.

23. Boyle CC, Kuhlman KR, Dooley LN, Haydon MD, Robles TF, Ang Y-S, et al. Inflammation and dimensions of reward processing following exposure to the influenza vaccine. Psychoneuroendocrinology. 2019;102:16-23.

24. Felger JC, Treadway MT. Inflammation effects on motivation and motor activity: role of dopamine. Neuropsychopharmacology. 2017;42:216-41.

25. Irwin MR, Olmstead R, Carroll JE. Sleep disturbance, sleep duration, and inflammation: a systematic review and meta-analysis of cohort studies and experimental sleep deprivation. Biol Psychiatry. 2016;80:40-52.

26. Kruse JL, Congdon E, Olmstead R, Njau S, Breen EC, Narr KL, et al. Inflammation and improvement of depression following electroconvulsive therapy in treatment-resistant depression. J Clin Psychiatry. 2018;79:17m11597.

27. Hodes GE, Pfau ML, Leboeuf M, Golden SA, Christoffel DJ, Bregman D, et al. Individual differences in the peripheral immune system promote resilience versus susceptibility to social stress. Proc Natl Acad Sci. 2014;111:16136-41.

28. Treadway MT, Buckholtz JW, Schwartzman AN, Lambert WE, Zald DH. Worth the "EEfRT?" The Effort Expenditure for Rewards Task as an objective measure of motivation and anhedonia. PLoS ONE. 2009;4:e6598.

29. Moieni M, Tan KM, Inagaki TK, Muscatell KA, Dutcher JM, Jevtic I, et al. Sex differences in the relationship between inflammation and reward sensitivity: a randomized controlled trial of endotoxin. Biol Psychiatry Cogn Neurosci Neuroimaging. 2019;4:619-26.

30. Lacourt TE, Vichaya EG, Escalante C, Manzullo EF, Gunn B, Hess KR, et al. An effort expenditure perspective on cancer-related fatigue. Psychoneuroendocrinology. 2018;96:109-17.

31. Treadway MT, Bossaller NA, Shelton RC, Zald DH. Effort-based decision-making in major depressive disorder: a translational model of motivational anhedonia. J Abnorm Psychol. 2012;121:553-8. https://doi.org/10.1037/a0028813.

32. Baglioni C, Spiegelhalder K, Lombardo C, Riemann D. Sleep and emotions: a focus on insomnia. Sleep Med Rev. 2010;14:227-38.

33. Lasselin J, Treadway MT, Lacourt TE, Soop A, Olsson MJ, Karshikoff B, et al. Lipopolysaccharide alters motivated behavior in a monetary reward task: a randomized trial. Neuropsychopharmacology. 2017;42:801-10. 
34. Gujar N, Yoo S-S, Hu P, Walker MP. Sleep deprivation amplifies reactivity of brain reward networks, biasing the appraisal of positive emotional experiences. J Neurosci. 2011;31:4466-74.

35. Mullin BC, Phillips ML, Siegle GJ, Buysse DJ, Forbes EE, Franzen PL. Sleep deprivation amplifies striatal activation to monetary reward. Psychol Med. 2013;43:2215-25.

36. Casement MD, Keenan $\mathrm{KE}$, Hipwell AE, Guyer AE, Forbes EE. Neural reward processing mediates the relationship between insomnia symptoms and depression in adolescence. Sleep. 2016;39:439-47.

37. Holm SM, Forbes EE, Ryan ND, Phillips ML, Tarr JA, Dahl RE. reward-related brain function and sleep in pre/early pubertal and mid/late pubertal adolescents. J Adolesc Health. 2009;45:326-34.

38. Irwin MR, Opp MR. Sleep health: reciprocal regulation of sleep and innate immunity. Neuropsychopharmacology. 2017;42:129-55.

39. Felger JC, Li Z, Haroon E, Woolwine BJ, Jung MY, Hu X, et al. Inflammation is associated with decreased functional connectivity within corticostriatal reward circuitry in depression. Mol Psychiatry. 2015;21:1358-65.

40. Vogelzangs N, Duivis HE, Beekman AT, Kluft C, Neuteboom J, Hoogendijk W, et al. Association of depressive disorders, depression characteristics and antidepressant medication with inflammation. Transl Psychiatry. 2012;2:79.

41. Beydoun MA, Obhi HK, Weiss J, Canas JA, Beydoun HA, Evans MK, et al. Systemic inflammation is associated with depressive symptoms differentially by sex and race: a longitudinal study of urban adults. Mol Psychiatry. 2019;16:22.
42. Carstensen LL. The influence of a sense of time on human development. Science. 2006;312:1913-5. https://doi.org/10.1126/science.1127488.

43. Rademacher L, Salama A, Gründer G, Spreckelmeyer KN. Differential patterns of nucleus accumbens activation during anticipation of monetary and social reward in young and older adults. Soc Cogn Affect Neurosci. 2014;9:825-31.

44. Segel-Karpas D, Ayalon L, Lachman ME. Retirement and depressive symptoms: a 10-year cross-lagged analysis. Psychiatry Res. 2018;269:565-70.

45. Noh J-W, Kwon YD, Lee L, Oh I-H, Kim J. Gender differences in the impact of retirement on depressive symptoms among middle-aged and older adults: a propensity score matching approach. PLoS ONE. 2019;14:e0212607.

46. Kendler KS, Gardner CO. Sex differences in the pathways to major depression: a study of opposite-sex twin pairs. Am J Psychiatry. 2014;171:426-35.

47. Borland JM, Aiani LM, Norvelle A, Grantham KN, O'Laughlin K, Terranova Jl, et al. Sex-dependent regulation of social reward by oxytocin receptors in the ventral tegmental area. Neuropsychopharmacology. 2019;44:785-92.

48. Eppinger B, Hämmerer D, Li S-C. Neuromodulation of reward-based learning and decision making in human aging. Ann NY Acad Sci. 2011;1235:1-17.

49. Spaniol J, Bowen HJ, Wegier P, Grady C. Neural responses to monetary incentives in younger and older adults. Brain Res. 2015;1612:70-82.

50. Felger JC, Haroon E, Patel TA, Goldsmith DR, Wommack EC, Woolwine BJ, et al. What does plasma CRP tell us about peripheral and central inflammation in depression? Mol Psychiatry. 2018. https://doi.org/10.1038/s41380-018-0096-3. 